\title{
Educación financiera en América Latina
}

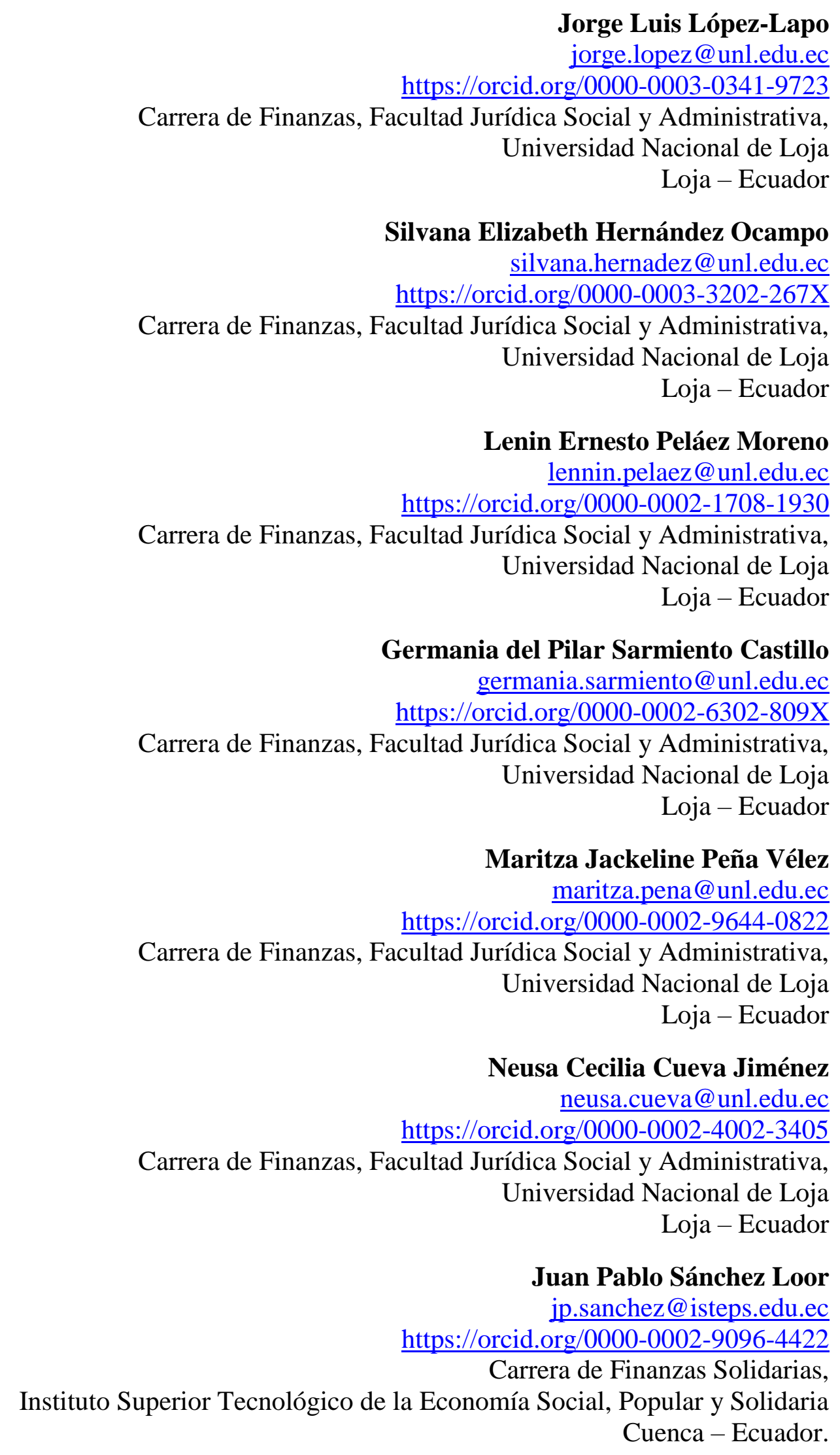




\section{RESUMEN}

La educación financiera es fundamental para la gestión de las finanzas personales. En este contexto, los autores proponen una revisión descriptiva de los programas de educación financiera en América Latina a fin de visualizar el alcance y resultados de cada uno de ellos. Se destacan las experiencias de Chile, México y otros países latinoamericanos, y el aporte de los programas de educación financiera al mejoramiento de la gestión de las finanzas personales como en la inclusión financiera. Sin embargo, también se vislumbran algunos retos vinculados a la estructura y gestión de los sistemas financieros latinoamericanos que generan barreras de acceso a sus servicios en especial para los segmentos más desfavorecidos de estos países. En sus conclusiones, los autores destacan que en general los programas de educación financiera han alcanzado resultados positivos, pero se han visto limitados por la estructura económica institucional de cada país.

Palabras clave: educación financiera; inclusión financiera; instituciones financieras; finanzas personales; latinoamérica. 


\title{
Financial education in Latin America
}

\begin{abstract}
Financial education is fundamental for the management of personal finances. In this context, the authors propose a descriptive review of financial education programmes in Latin America in order to visualise the scope and results of each of them. The experiences of Chile, Mexico and other Latin American countries are highlighted, as well as the contribution of financial education programmes to improving personal financial management and financial inclusion. However, some challenges linked to the structure and management of Latin American financial systems that generate barriers to access to their services, especially for the most disadvantaged segments of these countries, are also highlighted. In their conclusions, the authors highlight that in general, financial education programmes have achieved positive results, but have been limited by the economic and institutional structure of each country.
\end{abstract}

Keywords: financial education; financial inclusion; financial institutions; personal finance; Latin America.

Artículo recibido: 15 febrero 2022 Aceptado para publicación: 18 febrero 2022 Correspondencia: jorge.lopez@unl.edu.ec Conflictos de Interés: Ninguna que declarar 


\section{INTRODUCCIÓN}

En la última década, la mayoría de las economías de América Latina y el Caribe han mostrado un crecimiento sostenido que ha dado como resultado un incremento de su clase media (García et al., 2013). A pesar de estos avances, los niveles de pobreza y desigualdad siguen siendo altos y la exclusión financiera continúa afectando a sectores, tanto de la población urbana como rural, lo que puede dificultar el futuro desarrollo económico y social de la región.

El crecimiento económico trae consigo la necesidad de que las personas conozcan cómo manejar sus finanzas personales y beneficiarse de los mercados financieros más desarrollados. En este sentido, las iniciativas de educación financiera pueden convertirse en un complemento importante de los procesos de inclusión financiera y las medidas de reducción de la pobreza.

En efecto, García et al. (2013) define a la educación financiera como el proceso a través del cual consumidores e inversionistas mejoran su entendimiento sobre conceptos y productos financieros; y mediante la información, instrucción y orientación objetiva, desarrollan habilidades y la confianza para ser más conscientes de los riesgos y oportunidades financieras que les permitan tomar decisiones informadas, saber a dónde recurrir por ayuda y tomar otras medidas efectivas para mejorar su bienestar y seguridad. De ahí que los gobiernos de Latinoamérica han desarrollado programas que sirvan de complemento entre la educación financiera, la inclusión y el desarrollo del capital social. Lastimosamente las necesidades en materia de educación financiera de la población no son iguales para todos los países de la región, por lo cual es necesario un mayor esfuerzo en términos de medición. Esto, junto con una evaluación de impacto más completa de los programas existentes, ayudaría a diseñar e implementar programas de educación financiera más eficaces y a generar resultados medibles.

De acuerdo a Rivera y Bernal (2018) establecen que la educación financiera es una combinación de conocimientos, hábitos y actitudes que, si se practican de forma habitual en las decisiones económicas que se toman día con día, influyen positivamente en mejorar las finanzas personales, la economía y la calidad de vida de las personas que la practican. La educación financiera ha adquirido mayor relevancia como consecuencia de las situaciones adversas que afectan el crecimiento económico del país y la sociedad. 
$\mathrm{Al}$ respecto, en un estudio conjunto de la OCDE, CAF y CEPAL (2018) señalan que el $38,2 \%$ de la población latinoamericana no posee una cuenta y no conoce los requisitos para abrir una, adicional a ello menciona que un 14,3\% de estas personas no confían en las entidades financieras y que un $13,6 \%$ no le encuentra algún tipo de beneficio. Sobre estos mismos datos se pone de manifiesto que la bancarización es más proclive en zonas urbanas que en zonas rurales y que las mujeres son el grupo mayoritariamente excluido. Es importante destacar que, para Aguilar et al. (2019) el análisis de la implementación de programas de educación financiera, ayudaron a modificar en los estudiantes el patrón de gastos y comportamiento.

La educación financiera es un concepto dinámico y amplio que tiene diferentes elementos; no se trata solo de un conjunto de conocimientos específicos en un momento dado, sino de un flujo de formación, información y capacidad de los individuos para gestionar esa información a través de un proceso a medio y largo plazo (Instituto de Estudios Peruanos, 2016).

Esta investigación plantea acopiar de entre las mejores prácticas implementadas en Latinoamérica sobre programas de educación financiera, y conocer ¿cuál ha sido el impacto generado en cada uno de sus países?

\section{ESTRATEGIAS METODOLÓGICAS O MATERIALES Y MÉTODOS}

El desarrollo de la revisión bibliográfica referente a los programas de educación financiera en América Latina es de carácter descriptivo, teniendo como fin proporcionar al lector una visión sobre los programas de educación financiera desarrollados en América Latina y su incidencia en la población.

El fin de este tipo de revisión es proporcionar al lector una puesta al día sobre conceptos útiles en áreas de constante evolución. Se consideraron fuentes primarias y secundarias, con una validez de los últimos diez años.

Se partió de los fundamentos teóricos, para posteriormente caracterizar los programas de educación financiera en América Latina, con el fin de encontrar elementos comunes y diferenciadores entre ellos, finalmente se presentan las conclusiones que emanan de esta revisión.

\section{RESULTADOS Y DISCUSIÓN}

La perdurabilidad y la profundización de la desigualdad social en América Latina es un tema preocupante; varios estudiosos, líderes y gobernantes buscan la forma de superarla. 
Sin embargo, los avances son limitados y más bien las brechas entre pobres y ricos se amplían. Como resultado, los sectores rurales en especial los pequeños productores son los que con su esfuerzo producen alimentos a bajo costo y es la base para que los salarios de los trabajadores se mantengan en niveles mínimos; lo que posibilita la ampliación de las ganancias de los intermediarios y grandes empresas que son siempre las más beneficiadas originando un desface social. Entonces surge con fuerza el interés de encontrar políticas orientadas a mejorar la distribución de la riqueza para disminuir la pobreza y la exclusión social.

El acceso a una educación de calidad ofrece una vía de liberación de la pobreza y fomenta la realización de los derechos básicos. Tiene el poder de transformar vidas constituyéndose en un efecto igualador en la sociedad; todos los países deben tener como prioridad la pronta inversión en una educación pública, universal y de calidad que posibiliten una adecuada comprensión y construcción de una sociedad justa mediante la eliminación de barreras que originan la desigualdad y la exclusión (OXFAM, 2019). En relación a este ámbito, la presente investigación expone los resultados que explican la incidencia de los programas de Educación Financiera en la reducción de la pobreza y la desigualdad en América Latina.

Como resultado, la Educación Financiera hoy en día, es reconocida como tema económico fundamental por organizaciones internacionales como: el Banco Mundial, la Organización para las Naciones Unidas (UNICEF) y la UNESCO han advertido la necesidad de desarrollar y profundizar las capacidades financieras de la población mundial. Por lo que, la Educación Financiera ha comenzado a ser reconocida por los hacedores de las políticas públicas de los países latinoamericanos, como un componente importante para incidir sobre la dinámica económica del crecimiento.

Al respecto, para Dabla et al. (2015) la inclusión financiera alberga la promesa de fomentar el crecimiento y de reducir la pobreza y la desigualdad, en especial movilizando los ahorros y brindando a los sectores de hogares y de empresas mayor acceso a los recursos necesarios para financiar el consumo y la inversión. No obstante, Ardila y Rengifo (2014) señalan que el interés de tan solo propiciar crecimiento económico, no se constituye en garantía para superar la desigualdad y la pobreza.

En esta línea de pensamiento, en América Latina la Educación Financiera, es considerada como un tema prioritario en la agenda pública que debe ser tratado mediante la generación 
de políticas y la implementación de programas avalados por los entes educativos de cada país (ASOBANCARIA, 2016).

Igualmente, con la finalidad de promover la inclusión financiera, se han desarrollado una serie de iniciativas destinadas a aumentar los niveles de Educación Financiera de la población, siendo considerada como un proceso mediante el cual los usuarios comprenden mejor los productos y riesgos financieros, desarrollando habilidades para tomar decisiones mejor informadas con los riesgos que estas decisiones acarrean (García et al., 2013). Lamentablemente, esto no pasa de las buenas intenciones, los sectores populares continúan desenvolviéndose en la informalidad financiera.

En este sentido, en América Latina se evidencia que la mayoría de programas y modelos se desarrolla el primer principio (concienciación financiera) y de manera aún incipiente el segundo (desarrollo de las capacidades financieras), los cuales hacen énfasis en el acceso de información de los consumidores sobre los servicios financieros y la enseñanza y asesoría frente a la toma de decisiones informadas en pro del bienestar y el desarrollo de una comprensión financiera más consciente.

Los países de Latinoamérica han enfocado sus programas de educación financiera en la concienciación de la importancia del saber financiero; en los objetivos de los programas, el común denominador es la enseñanza de conceptos financieros, tales como el ahorro, la inversión, el costo de los préstamos, en pocos casos el objetivo de los programas ha sido incentivar emprendimientos con preparación financiera (Aguilar, Carvajal y Serrano, 2019). Se podría afirmar que la inclusión financiera a través del apoyo a los pequeños y medianos emprendimientos no se concreta por los altos costos, cortos plazos y fundamentalmente por no ser considerados como sujetos de crédito, con lo que se reafirma la exclusión.

De ahí que, en América Latina, se observe un bajo nivel de comprensión de conceptos financieros básicos como: inflación, tasa de interés, relación entre riesgo y rentabilidad, y funcionamiento del mercado de capitales. Por este motivo, García et al. (2013) señala que la prioridad de formación debería estar dirigida a la comprensión y asesoramiento de los servicios financieros, enfatizando en conceptos como el ahorro y el crédito, los cuales contribuyen a la planificación financiera de largo plazo (García et al., 2013).

Por consiguiente, el objetivo es superar el analfabetismo financiero en la población, pues a partir de varios estudios previos se identificó que hay un gran margen de población que 
no comprende en su totalidad la manera efectiva de utilizar los servicios y productos financieros, lo que provoca una toma de decisiones sin la información apropiada (Ardila y Rengifo, 2014). Si a esta realidad identificada como analfabetismo financiero, se le agrega la abultada y engorrosa tramitología que se requiere para acceder a un crédito; estas exigencias inaccesibles se las encuentra tanto en la Banca Pública como la Privada, esto explica por qué los sectores populares prefieren la informalidad e incluso el agiotismo para obtener recursos financieros para sus emprendimientos.

En América Latina, esto podría cambiar si el punto de partida fuera el reconocimiento de las necesidades específicas de la población: sus actividades productivas y sus formas de intercambio monetarias y no monetarias. Es así que para cubrir estas necesidades se desarrollan modelos y programas de Educación Financiera orientados a diversas poblaciones de acuerdo a sus condiciones sociales y económicas, cuyo propósito es el fortalecimiento de capacidades financieras de la población de bajos recursos.

La adopción de dichos programas y modelos se establece por medio de políticas gubernamentales que se apoyan en los enunciados de Educación Financiera que propone la OCDE, los cuales cada país apropia teniendo en cuenta las condiciones particulares que los caracteriza. Otro aspecto a considerar por los programas de educación financiera de América Latina es que puedan estar enfocados tanto para niños como para adultos, a través del uso de talleres interactivos, videos, fotografías y folletos que tienen como finalidad incentivar la cultura del ahorro en todos los participantes, lo que a largo plazo puede dar como resultado un crecimiento económico o productivo. Esta perspectiva de ser sostenida podría provocar cambios sostenibles en la Educación Financiera en América Latina.

De tal forma, se puede destacar que los beneficios de la inclusión financiera podrían ser particularmente importantes en América Latina y el Caribe donde el crecimiento es modesto y volátil, la pobreza y la desigualdad siguen siendo ingentes, la inversión y el ahorro son bajos y la informalidad está sin control. Sin embargo, esta promesa debería considerar políticas que coadyuven a la sostenibilidad de pequeños y medianos emprendimientos en especial a los que ofrecen bienes y servicios de consumo masivo como son los alimentos y el vestido. 


\section{Programas de Educación Financiera dentro de las políticas públicas de los gobiernos de América Latina}

Siguiendo el ejemplo del gobierno de Estados Unidos de América y la Comisión Europea (2007), con respecto a la importancia de planificar, ejecutar y poner en marcha programas de educación financiera, que desarrollan competencias necesarias para mejorar la cultura financiera de sus conciudadanos, los países de América Latina han implementado programas de alfabetización financiera. Dichos programas abordan no solo temas financieros básicos, sino también los servicios financieros cada vez más complejos, con lo cual se esperan que sus ciudadanos puedan tomar mejores decisiones financieras según sus circunstancias particulares.

En este contexto, García et al. (2013) mide los conocimientos y capacidades financieras en ocho países de la región, lo cual le permite evaluar las políticas implementadas en cuanto a la eficacia de los programas financieros implementados a los ciudadanos por cada gobierno a través de los organismos especializados de: Argentina, Brasil, Bolivia, Chile, Ecuador, Paraguay, Perú y Colombia. Los cuestionarios abordan la capacidad financiera y miden habilidades, actitudes y comportamientos de los individuos mediante encuestas que tratan temas financieros.

Algunos resultados se muestran a continuación con respecto a las actitudes y comportamientos financieros. Durante 2019 y 2020 se obtuvo el promedio de cuatro países: Brasil, Perú, Colombia y Ecuador y relacionado al porcentaje de ahorros, se determinó que el $61 \%$ de las personas no ahorran y en caso de hacerlo, el ahorro es informal. Brasil en este parámetro aportó con un porcentaje mayor que es del $72 \%$ de ahorro formal en cuentas del sistema bancario del país. Además, el 57\% de la población de los países mencionados no tienen la capacidad de cubrir gastos imprevistos que se relacionen con el ingreso promedio percibido.

El 75\% de ecuatorianos, peruanos, brasileños y colombianos en promedio, no tienen una meta financiera. Otro dato importante es que, la población de Ecuador tiende más a buscar fuentes adicionales de ingresos a diferencia de la mayoría de la población de Brasil que no tiene un plan de acción en cuanto a nuevas formas de generar ingresos.

Los planes de jubilación o pensión son un tema que Brasil lleva la delantera, debido a que, el $34 \%$ de su población con características de ser beneficiario, se encuentra amparada por un plan de jubilación, seguido por $32 \%$ de colombianos, $31 \%$ de ecuatorianos y $21 \%$ 
de peruanos que se sienten muy seguros debido a las pensiones que reciben. En este punto es importante analizar que las personas en edad para recibir una pensión por jubilación, en su mayoría deben seguir trabajando para suplir este beneficio, al cual el $85 \%$ en el caso de Ecuador no lo tienen, así mismo el 61\% en Brasil, 42\% en Colombia y 35\% en Perú. En lo que respecta a la medición de niveles de conocimientos financieros de la población de los cuatro países, se obtuvo como hallazgo que el $50 \%$ de peruanos y colombianos conocen el efecto de la inflación en el poder adquisitivo de la moneda a diferencia del $33 \%$ de ecuatorianos y la población de Brasil que conocen sobre este tema. En cuanto al conocimiento básico de una tasa de interés simple, los porcentajes promedios sobre este tema son desalentadores, tan solo el 22\% en Perú, $19 \%$ en Ecuador, 13\% en Brasil y 10\% en Colombia la población es conocedora de su cálculo, significancia e importancia.

Los resultados sobre la encuesta de capacidades financieras aplicada por la CAF, permite a los gobiernos la implementación de mejoras en la consecución de los planes de capacitación y educación financiera, que desarrollen una verdadera cultura financiera acorde con las necesidades de cada contexto poblacional y que tenga en cuenta las diferencias de los segmentos poblacionales dentro de cada país.

\section{Nexo entre el ingreso, ahorro y consumo como argumentos válidos en la educación financiera}

Como plantean Lusardi y Mitchell (2014) la educación financiera enfocada a los conocimientos en prácticas de ahorro y consumo permite que, un individuo bien informado consuma menos que sus ingresos en las épocas cuando estos últimos se incrementan, concretándose una práctica de ahorro destinada a solventar el consumo cuando justamente su renta ha bajado, como es el caso de ingresos por jubilación. En microeconomía estas prácticas se refieren a tasa de aversión al riesgo, tasa de descuento, restricciones de liquidez, restricciones sociales, entre otras.

Surgen así entonces modelos microeconómicos que sustancialmente superan a los conocimientos básicos sobre educación financiera, de ahí la importancia que las instituciones ligadas al sistema financiero, con palabras y expresiones articuladas de forma clara y concisa, expliquen al consumidor que en base a estos modelos microeconómicos se han postulado productos y servicios financieros como planes de ahorro y reducción de gastos. La armoniosa concepción de los temas consiente formular la oferta, que en el mediano plazo les va a permitir a la población de interés conseguir un 
monto determinado en el caso del ahorro, sin embargo, son pocas las personas que conocen y comprenden estos cálculos económicos, más bien les parecen complejos, lo que hace es restar importancia, prestar poca atención y surge una brecha entre el modelado y la realidad a explorar.

De este modo, se ve la necesidad de aportar con la difusión de la teoría financiera que enriquece los conocimientos y deja una ruta abierta a la política de las instituciones financieras que consigue orientarse de mejor manera a la consecución de sus objetivos dentro del mercado, y las personas logran en entendimiento óptimo en aspectos como: identificación de activos con mejor rendimiento, propósito de productos y transacciones más complejas como la adquisición de bonos.

Por otro lado los autores referidos inicialmente, hacen notar que la educación financiera o alfabetización financiera debería incorporar en el avance teórico de la impartición de conocimientos, temas concernientes a las situaciones tensas que se originan por los préstamos o créditos bancarios ocasionando trances en la salud que puede llevar a un riesgo alto de mortalidad, no sin restar importancia a las dificultades intrafamiliares que el desacuerdo en inversiones y consumo sin fundamento lógico financiero pueden suscitarse.

\section{Impacto de los programas de educación financiera en Chile}

Chile luego de la crisis suprime suscitada en 2008, ha tomado muy en serio ubicar en sus políticas gubernamentales de educación, temáticas relacionadas a la educación financiera como una estrategia que genera beneficios sociales, abordando no solamente el acceso y uso de productos financieros, sino también acerca de la importancia del respeto y cumplimiento de leyes que regulan y protegen los procesos en esta industria desde su inicial inclusión hasta las grandes negociaciones en mercado financieros.

De similar forma, se precisa que los habitantes del país requieran del conocimiento del uso de herramientas que les permitan considerar las mejores alternativas de financiamiento, ahorro e inversión considerando la profundización y bancarización a la que las personas acceden con mayor facilidad.

El Servicio Nacional del Consumidor (SERNAC) de Chile en 2021 lleva un programa de Educación Financiera dirigido a los jóvenes de una edad comprendida entre 18 y 29 años y es abordado en 5 módulos: introducción, créditos, endeudamiento juvenil, derechos del consumidor y planificación presupuestaria; mientras que la Universidad Autónoma de 
Chile en la carrera de Derecho en su currículo, presenta cierto compendio de asignaturas relacionadas al tema tratado, éstas son: Derecho Económico, Derecho de Sociedades, Introducción a la Economía, Reorganización y Liquidación Patrimonial.

Los resultados en la comparación de los programas de Educación Financiera de la SERNAC y la Universidad Autónoma de Chile, muestran que la institución estatal incluye la enseñanza del consumo responsable, temas sobre crédito, tipos, costo, valores de cuotas, endeudamiento, sobreendeudamiento, tópicos que los estudiantes universitarios no los estudian, sin embargo, su malla curricular si proporciona los conceptos relacionados a procesos en las que las empresas y personas naturales participan como es la insolvencia y cesación de pagos (Bozzo y Remeseiro, 2021).

En un nivel de 1 a 7, se evaluó el conocimiento promedio de los estudiantes universitarios autónomos en Chile con respecto a la formación financiera, estableciéndose tres rangos de dificultad en las interrogantes con lo cual se obtuvo los siguientes resultados: preguntas con dificultad alta el promedio fue de 3,81; dificultad media arrojó un 3,8 y dificultad baja se evidenció el promedio de 4,6 puntos.

El estudio llega a dos conclusiones, estas son: 1) si bien los estudiantes tienen conocimientos jurídicos de herramientas financieras, estos no son lo suficientemente significativos, para poseer competencias que les permitan tomar decisiones correctas en lo que respecta al comportamiento adecuado como consumidor y 2) el programa de educación financiera llevado a cabo por la SERNAC, institución pública, debe servir de modelo e inspiración en la planificación de currículos universitarios y fortalecer los ya existentes, en los que se entregue formación útil en la práctica, es decir, reformularlos con un enfoque menos jurídico que el que se está llevando y optar por solventar la falta de conocimientos financieros mediante la articulación de un módulo que abarque en su totalidad esta materia con carácter práctico, interactivo, ejemplificativo y pertinente a la realidad vital de los beneficiarios, en este caso los alumnos universitarios.

\section{La Educación Financiera en México y otros países de la OCDE}

En México, a pesar de los esfuerzos orientados hacia una mayor difusión de la educación financiera, todavía se detectan importantes deficiencias que pueden perjudicar el bienestar de las personas y de sus familias, por ello es importante fomentar la inclusión de todos los estratos sociales. 
El estudio que se detalla en esta sección es abordado por Mungaray, González, y Osorio (2021) quienes llegan a conclusiones varias, entre ellas que el crecimiento económico de México no es sostenido debido a la falta de educación financiera en la población. En lo concerniente a la evaluación de conocimientos relacionados a temas financieros, mediante la metodología usada por la Organización para la Cooperación y el Desarrollo Económicos que considera al conocimiento, actitudes y comportamiento como indicadores explicativos dentro de la Encuesta Nacional de Inclusión Financiera de la Comisión Nacional Bancaria y de Valores.

La investigación durante 2018 encontró los siguientes hallazgos, para el caso de México con una muestra de 12.446 participantes, los resultados fueron comparados con los estudios de la OCDE efectuados en el 2017. Los valores son los siguientes:

1) Con valores normalizados para 2017 la OCDE encontró que el promedio de conocimientos acertados en la población mexicana es del 57,62\% con relación al promedio del $60.74 \%$ que obtienen los países del G20 ubicándose México en el puesto No. 12. Posteriormente se evidenció que, a pesar de la gestión realizada por la Secretaría de Hacienda y Crédito Público, en cuanto a la impartición y promoción de temas financieros entre la población, el estudio estimó que en el 2018 los resultados son igualmente desalentadores, ya que el promedio en conocimientos financieros se incrementó tan solo el 1\%, es decir llegó al 58,63\%.

2) Del resultado anterior se desprende que, los factores comportamiento y actitudes financieras inclusive aportan en menor porcentaje al promedio del año 2018 de conocimientos financieros generales.

3) En la pesquisa de evaluación con técnicas econométricas, el modelo estimó que en promedio si una persona incrementa sus conocimientos en el área financiera en $1 \%$, sus ingresos se verán incrementados en el $1.75 \%$, esto es interpretado además como una mayor inclusión social que relaciona directamente las variables ingresos y conocimientos financieros.

Seguidamente, la investigación que refiere al estudio del conocimiento financiero en 32 países de la OCDE descrito en el trabajo de Oliver, Amate y Guarnido (2017) indica que los hallazgos fueron similares al descrito en el apartado anterior y relaciona a la equidad económica como una variable efecto del incremento en el conocimiento financiero de la población, esta permite reducir la desigualdad en la distribución de la renta a pesar de que 
los inversores en los casos estudiados estuvieran protegidos por normativas y leyes gubernamentales.

Los hallazgos también destacan que cuando el ahorro se incrementa en mayor tasa que la del crecimiento poblacional, en la mayoría de países miembros de la OCDE, en el largo plazo se produce un ingente crecimiento en el stock de capital, situación que directamente es el principio de la perturbación en la distribución equitativa de la riqueza, sin embargo, no siempre la propagación de productos financieros en la línea de decisiones adecuadas de inversión llevan a la desigualdad en la distribución de la renta, por esta razón la política gubernamental de los países es de dotar a su población de mayores conocimientos financieros como mecanismo agregado de la equidad económica.

\section{Ejecución de programas financieros en Argentina y Uruguay}

De la revisión de literatura sobre los programas de Educación Financiera en Argentina, Bolivia, Paraguay, Perú y Uruguay, se encontró como hallazgos que, están dirigidos a la población de temprana edad, jóvenes y adultos mediante procesos de capacitación que involucran la ejecución de talleres interactivos y la revisión de material acorde a conocimientos generales que poseen previamente, con la finalidad de que, los participantes en el mediano y largo plazo, puedan manejar sus recursos financieros adecuadamente mejorando su productividad y logrando el crecimiento económico (Aguilar, Carvajal y Serrano, 2019).

Educación financiera en Argentina, a partir de 2019 se desarrolla el Plan Nacional de Educación Financiera (PNEF) que tiene como objetivo mejorar las capacidades y conocimientos financieros de manera responsable de los individuos y empresas, promoviendo el acceso y uso de servicios financieros. Fundamentándose en que el país registra un porcentaje menor de actitudes financieras adecuadas en comparación al promedio de los países del G20 y en general el índice de educación financiera en conocimientos, comportamientos y actitudes financieras posiciona a la Argentina en el puesto 37 de 39 en total con 11,5 puntos, de este modo, el PNEF tiene una vigencia al año 2023 en el cual se establece como meta mejorar este indicador.

Las acciones estratégicas planteadas por el gobierno argentino en el PNEF (2019) entre otras son que en las escuelas secundarias se incorpore contenidos de educación financiera, minimizar actividades abusivas y fraudulentas, potenciar el conocimiento y acceso a servicios financieros prefiriendo los formales a informales, fomentar la oferta responsable 
de créditos y proteger al consumidor mediante la difusión de campañas e impartición de conocimientos relacionados al consumo responsable.

Educación financiera en Uruguay, desde el año 2012 el Banco Central de Uruguay viene desarrollando el programa BCUEduca que se refiere al programa de Educación Económica y financiera con el objetivo de promover y desarrollar la educación y cultura económica y financiera de la población uruguaya con actividades como ferias interactivas de Economía y Finanzas, talleres y cursos. Entre las principales acciones se destacan los cursos dirigidos a cuatro grupos en que la toma de decisiones incide en la colectividad: periodistas, trabajadores $\mathrm{y}$ familias, dirigentes sindicales, docentes de primaria $\mathrm{y}$ secundaria y estudiantes de bachillerato.

Uruguay tiene claro que los grupos a los que se destina la capacitación en temas financieros se fundamenta en teorías modernas llamadas "economía de la conducta" pues se estima que las decisiones personales son fundamentales para la construcción de la sociedad en aprovechar mejor el conocimiento transmitido para organizar las finanzas personales y familiares. Así mismo, destaca que no se esperan resultados positivos consecuencia de la educación financiera sin una inclusión financiera.

La base de datos Global Findex 2017 destaca los resultados de los programas de educación financiera en Uruguay y atribuye a estos que el país es el que más incremento en el acceso a instrumentos financieros ha logrado en la región a partir del año 2011; de esta forma el porcentaje de adultos que accedieron a instrumentos financieros se incrementó de 25\% en 2011 al 64\% en 2017 (Demirgüç, Klapper, Singer, Ansar y Hess, 2018).

\section{CONCLUSIÓN O CONSIDERACIONES FINALES}

La importancia de la educación financiera ha sido resaltada en todos los casos estudiados, coincidiendo en la necesidad de desarrollar las capacidades financieras para promover no solo el desarrollo personal sino también aportar al crecimiento económico de cada país. Sin embargo, se observa también debilidades en los programas de educación financiera, los cuales en gran parte se dedican a describir un programa curricular estrictamente teórico, y con un limitado impacto en las capacidades financieras prácticas. A esto se suma que los programas de educación financiera se enfrentan a cuestiones como el limitado acceso al sistema financiero, y problemáticas económicas sociales, de exclusión y pobreza. Los resultados muestran que la educación financiera puede aportar de forma 
limitada al mejoramiento de la calidad de vida de los sectores más vulnerables, volviéndose necesario una integración estructural entre la educación financiera y las políticas estatales.

En síntesis, los programas de educación financiera de la región han aportado principalmente a conocimiento teórico de las finanzas, pero no han logrado integrarlos en habilidades financieras prácticas. Ante esto, se vuelve necesario rediseñar los programas de educación financiera e integrarlos en una política pública más amplia que abarque también soluciones a las problemáticas de pobreza y exclusión que existen en América Latina.

\section{Agradecimientos}

Este trabajo de investigación fue apoyado por la Universidad Nacional de Loja, en el ámbito del proyecto 40-DI-FJSA-2021.

\section{LISTA DE REFERENCIAS}

Aguilar, M., Carvajal, R. y Serrano M. (2019). Programas de educación financiera implementados en América Latina. Revista de Investigación, Formación y Desarrollo: Generando Productividad Institucional, 7(2), 72-88. https://doi.org/10.34070/rif.v7i1

Ardila, Y., y Rengifo, L. (2014). Aproximaciones a la Educación Financiera en América Latina. XIX Congreso Internacional de Contaduría, Administración e Informática. Obtenido de https://bit.ly/35mNtlF

ASOBANCARIA. (2016). Programas de Educación Financiera en Latinoamérica: un énfasis en el caso colombiano. Semana Económica, 1-13. Obtenido de https://bit.ly/3nHf7jE

Bozzo, S., y Remeseiro, R. (2021). Hacia un currículo que fortalezca la educación financiera en las carreras de Derecho. Revista Pedagogía Universitaria y Didáctica del Derecho, 8(1), 45-68. https://doi.org/10.5354/0719$\underline{5885.2021 .61454}$

Dabla, E., Deng, Y., Ivanova, A., Karpowicz, I., Unsal, F., VanLeemput, E., y Wong, J. (2015). Inclusión financiera: Un enfoque centrado en América Latina. IMF Working Paper, (15)206. Obtenido de https://bit.ly/3I1shzA 
Demirgüc, A., Klapper, L., Singer, D., Ansar, S., y Hess, J. (2018). La base de datos Global Findex 2017: Medición de la inclusión financiera y la revolución de la tecnología financiera. World Bank Group. Obtenido de https://bit.ly/3fT3npN

García, N., Grifoni, A., López, J., y Mejía, D. (2013). La educación financiera en América Latina y el Caribe: Situación actual y perspectivas. Serie políticas públicas y transformación productiva, 12. Obtenido de https://bit.ly/3K6oBym

Gobierno de la República Argentina. (2019) Plan Nacional de Educación Financiera Argentina 2019. Obtenido de https://bit.ly/3Fwl4WL

Instituto de Estudios Peruanos. (2016). Experiencias de educación financiera del Proyecto Capital en América Latina. En breve, 57. Obtenido de https://bit.ly/33vVOT7

Lusardi, A., y Mitchell, O. (2014). The economic importance of financial Literacy: Theory and evidence. Journal of economic Literature, 52(1), 5-44. http://dx.doi.org/10.1257/jel.52.1.5

Mungaray, A., González, N., y Osorio, G. (2021). Educación financiera y su efecto en el ingreso en México. Problemas del Desarrollo. Revista Latinoamericana de Economía, (52)205. https://doi.org/10.22201/iiec.20078951e.2021.205.69709

OCDE, CAF y CEPAL. (2018), Perspectivas económicas de América Latina 2018: repensando las instituciones para el desarrollo. Ediciones OCDE. París. http://dx.doi.org/10.1787/leo-2018-es

Oliver, F., Amate, I., y Guarnido, A. (2017). El conocimiento financiero y su impacto en la desigualdad de la renta. XXIV Encuentro de Economía Pública. España. Obtenido de https://bit.ly/3H1nlKS

OXFAM. (2019). El poder de la educación en la lucha contra la desigualdad. Oxfam Internacional. Obtenido de https://bit.ly/3rQ3urI

Ramos, J., y Calderón, F. (2011). Matriz de programas de educación financiera en América Latina y el mundo. En breve, 25. Obtenido de https://bit.ly/3A4kFd4

Rivera, B. y Bernal, D. (2018). La importancia de la educación financiera en la toma de decisiones de endeudamiento. Estudio de una sucursal de "Mi Banco" en México. Revista Perspectivas, (41), 117-144. Obtenido de https://bit.ly/3IDX8T1 